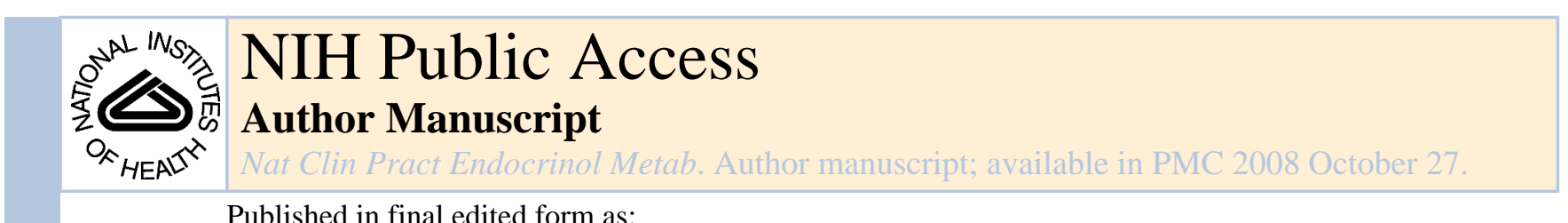

Published in final edited form as:

Nat Clin Pract Endocrinol Metab. 2008 July ; 4(7): 382-393. doi:10.1038/ncpendmet0843.

\title{
The prevention of type 2 diabetes
}

Jill P Crandall ${ }^{*}$, Associate Professor of Clinical Medicine at Albert Einstein College of Medicine, Bronx, NY.

William C Knowler,

Chief of the Diabetes Epidemiology and Clinical Research Section of the National Institute of

Diabetes and Digestive and Kidney Diseases, Phoenix, AZ.

Steven E Kahn,

Professor of Medicine at Veterans' Affairs Puget Sound Health Care System, Seattle, WA.

David Marrero,

JO Ritchey Professor of Medicine at Indiana University, IN.

Jose C Florez,

Assistant in Medicine at the Center for Human Genetic Research and Diabetes Unit, Massachusetts General Hospital, Boston, MA.

George A Bray, Professor of Medicine at Louisiana State University, Baton Rouge, LA.

Steven M Haffner,

Professor of Internal Medicine in the Departments of Medicine and Clinical Epidemiology, University of Texas Health Science Center, San Antonio, TX.

Mary Hoskin, and

Program Coordinator of the Diabetes Prevention Program Outcomes Study in the Diabetes Epidemiology and Clinical Research Section, National Institute of Diabetes, Digestive and Kidney Diseases, Phoenix, AZ.

David M Nathan

Director of the Diabetes Center and General Clinical Research Center in Harvard Medical School, Boston, MA, USA.

Diabetes Prevention Program Research Group

\section{SUMMARY}

Type 2 diabetes mellitus (T2DM) affects more than $7 \%$ of adults in the US and leads to substantial personal and economic burden. In prediabetic states insulin secretion and action-potential targets of preventive interventions-are impaired. In trials lifestyle modification (i.e. weight loss and exercise) has proven effective in preventing incident T2DM in high-risk groups, although weight

\footnotetext{
*Correspondence Diabetes Research Center, Albert Einstein College of Medicine, 1300 Morris Park Avenue, Bronx, NY 10461, USA crandall@aecom.yu.edu.

REVIEW CRITERIA

We searched MEDLINE and PubMed for original articles published between 1960 and 2007 that focused on type 2 diabetes prevention trials. The search terms used were "type 2 diabetes", "prevention", "impaired glucose tolerance" and "prediabetes". We included Englishlanguage, full-text articles that reported trials in which diagnosis of diabetes was a predefined study outcome. We also searched the reference lists of identified articles for additional papers and we sought expert opinion on relevant trials.

Competing interests

SE Kahn and SM Haffner have declared associations with the following companies: Astra Zeneca, GlaxoSmithKline, Merck, Novartis and Pfizer. See the article online for full details of the relationships. The other authors declared no competing interests.
} 
loss has the greatest effect. Various medications (e.g. metformin, thiazolidinediones and acarbose) can also prevent or delay T2DM. Whether diabetes-prevention strategies also ultimately prevent the development of diabetic vascular complications is unknown, but cardiovascular risk factors are favorably affected. Preventive strategies that can be implemented in routine clinical settings have been developed and evaluated. Widespread application has, however, been limited by local financial considerations, even though cost-effectiveness might be achieved at the population level.

\section{Keywords}

impaired glucose tolerance; prediabetes; prevention; type 2 diabetes

\section{INTRODUCTION}

Type 2 diabetes mellitus (T2DM) is rapidly becoming the most common chronic disease in the US, with more than $7 \%$ of the adult population affected and 1,500,000 new cases diagnosed per year. ${ }^{1}$ T2DM is most prevalent among the elderly and in specific ethnic groups, including African Americans, Hispanic Americans, Asian and Pacific Island Americans and American Indians. Diabetes is accompanied by a multitude of severe, long-term complications that ultimately cause more adult cases of blindness, renal failure and amputations in the US than any other disease. In addition, people with T2DM have a twofold to fourfold increase in their risk of developing cardiovascular and peripheral vascular disease and stroke. The enormous human and financial costs that accompany T2DM, and the challenge of treating it effectively once it has developed, make it an appropriate target for prevention. As reviewed here, substantial progress has been made in the past decade in developing and evaluating effective preventive strategies.

\section{PREVENTIVE STRATEGIES BASED ON PATHOPHYSIOLOGY}

T2DM is characterized by insulin resistance and $\beta$-cell dysfunction (impaired insulin secretion), the balance between which varies widely between individuals. ${ }^{2,3}$ Insulin resistance is frequently well established in individuals with impaired glucose tolerance, and it is the presence of $\beta$-cell dysfunction that distinguishes those in whom glucose intolerance worsens from those in whom it remains stable. ${ }^{4}$ Interventions that decrease insulin resistance and preserve or improve $\beta$-cell function are likely to be effective in slowing progression from impaired glucose tolerance to diabetes, or even allowing reversion to normal glucose tolerance. Many interventions, such as drugs, weight loss and physical activity, are thought to improve insulin resistance and might also affect insulin secretion.

\section{EARLY PREVENTION STUDIES}

Randomized clinical trials of preventive strategies for T2DM began in the 1960s, notably before the current definitions were established for impaired fasting glucose and impaired glucose tolerance - both of which indicate a high risk of T2DM. Three early trials examined drugs then in common use to treat T2DM. In the Whitehall study, 204 men with impaired glucose tolerance were randomly assigned either phenformin or placebo. ${ }^{5}$ In the 181 patients who completed 5 years of follow-up, the cumulative incidence of diabetes was $14 \%$ in the phenformin-treated patients and $16 \%$ in placebo-treated patients, with a cumulative incidence rate ratio (drug versus placebo) of 0.9 (95\% CI 0.4-1.8). ${ }^{6}$ In the Bedford study, 241 men and women with impaired glucose tolerance were randomly assigned either the sulfonylurea, tolbutamide, or placebo. The 8.5-year cumulative incidence of diabetes was $9 \%$ in the tolbutamide group and $8 \%$ in the placebo group (incidence rate ratio $1.1,95 \% \mathrm{CI} 0.5-2.5$ ) and a subsequent report of 10 years' follow-up of these patients also showed no significant effect of tolbutamide on the incidence of diabetes. ${ }^{7}$ The third major study of this era was conducted 
in 147 men in Malmöhus, Sweden. ${ }^{8}$ Men with impaired glucose tolerance (defined according to local criteria) received dietary advice to limit their carbohydrate and lipid intake and, if overweight, their total energy intake. They were also randomly assigned tolbutamide, placebo or no drug treatment. The 10-year cumulative incidence of diabetes was $10 \%$ in men assigned tolbutamide treatment and $13 \%$ in the two groups assigned placebo or no drug (incidence rate ratio $0.8,95 \%$ CI $0.3-2.0){ }^{6}$

None of these pioneering studies established whether diabetes could be prevented or delayed, and their findings were inconclusive owing to the small sample sizes used. Whether pharmacologic prevention of T2DM was possible remained unknown into the 1990s. The Malmöhus study, however, provided two additional provocative findings. The results of the study were originally reported according to patients' estimated adherence to drug treatment, rather than according to their original treatment assignment (i.e. by intention to treat). ${ }^{8}$ Of the few tolbutamide-treated participants who were estimated to have taken the drug throughout the study, however, none developed diabetes. This outcome has been widely cited as evidence that tolbutamide has the potential to prevent diabetes. This conclusion of the article was, however, inappropriately based on the analysis of adherence. Analysis by intention to treat, generally considered the proper way to assess treatment effects in a clinical trial, did not support a preventive effect of tolbutamide. In addition, although treatment and formal follow-up of the study participants ended in 1975, the investigators continued to follow up participants until 1987 for mortality and cause of death. The all-cause mortality rate ratio (drug compared with placebo or no drug) was 0.66 (95\% CI $0.39-1.10)$ and the ischemic heart disease mortality rate ratio was 0.42 (95\% CI $0.16-1.12){ }^{9}$

While the conclusions of this study were limited by its small size and wide CIs, the Malmöhus study provided early support for the hypothesis that the benefits of treatments for hyperglycemia might persist long after active intervention ends. This hypothesis has been supported by later, much larger clinical trials. ${ }^{10,11}$

\section{STUDIES OF LIFESTYLE MODIFICATION}

\section{Diet and exercise}

Over the past 10 years several randomized clinical trials have formally tested the hypothesis that lifestyle modification, namely weight loss and/or increased activity or exercise, can prevent T2DM. ${ }^{12}$ Their findings indicate that such changes can prevent the onset of diabetes in people at risk (Table 1).

The first of these studies was conducted in Chinese community health clinics and included 577 individuals with impaired glucose tolerance. ${ }^{13}$ Individuals were assigned, according to which clinic they attended, to a program of dietary modification, exercise, or both, and were followed up for 6 years. The dietary intervention focused on increased consumption of vegetables and reduced consumption of alcohol and simple carbohydrates; overweight individuals (BMI $\geq 25$ $\mathrm{kg} / \mathrm{m}^{2}$ ) were also advised to limit their energy intake. Participants in the exercise-only group were instructed to increase their daily activity by the equivalent of at least $20 \mathrm{~min}$ of brisk walking. The diet plus exercise group received both interventions, and patients who attended usual-care clinics served as a control group. The 6-year cumulative incidence of diabetes was high in all groups $(48 \%, 41 \%, 46 \%$ and $68 \%$ in the diet-only, exercise-only, diet plus exercise and control groups, respectively). The relationship between the amount of weight lost and diabetes incidence was inconsistent and all three interventions were similarly effective in preventing diabetes.

The Finnish Diabetes Prevention Study (DPS) ${ }^{14}$ was a randomized study of 522 over-weight, middle-aged adults with impaired glucose tolerance. The lifestyle intervention included dietary 
and exercise components. The weight-loss goal was $5 \%$ or more of baseline weight, and to achieve this target participants were instructed to reduce fat intake and increase consumption of fiber, whole grains, vegetables and low-fat dairy products. The exercise component required participants to do moderate-intensity exercise for at least $30 \mathrm{~min}$ per day. The intervention group lost an average of $4.2 \mathrm{~kg}$ in the first year of the study and, compared with the control group, had a 58\% reduction in diabetes incidence during the whole study ( $\sim$ years). Although this study was not designed to assess the individual contributions of the diet and exercise components, participants who undertook particularly vigorous leisure-time physical activities had a markedly reduced risk of diabetes. ${ }^{15}$ After cessation of the intervention, the reduction in diabetes risk persisted - over 7 years of follow-up, the intervention group had an overall reduction in their risk of diabetes of $43 \%$ compared with the control group; during the post intervention period, the risk reduction was $36 \%$ in the subset of participants who were free from diabetes at the end of the intervention period despite modest weight regain $(1.3 \mathrm{~kg}$ regained of the original $3.1 \mathrm{~kg}$ lost by this subgroup) in the intervention group. ${ }^{16} \mathrm{As}$ might be expected, individuals who maintained the changes to their lifestyle during the extended followup period had the greatest reduction in their risk of diabetes (a 46\% risk reduction compared with those who did not achieve their goals). In a multivariate analysis that considered the success of achieving all five lifestyle goals, only weight reduction remained a significant determinant of diabetes risk.

The largest and most comprehensive lifestyle modification study was the US Diabetes Prevention Program (DPP; for a list of members of the DPP Research Group see Supplementary information online). ${ }^{17,18}$ The DPP randomly allocated 3,234 overweight, mostly middle-aged adults with impaired glucose tolerance and fasting plasma glucose values of $5.3 \mathrm{mmol} / \mathrm{l}$ or higher to intensive lifestyle intervention, metformin or placebo. An additional 585 participants were randomly assigned troglitazone therapy, but recruitment to this group and participants' ongoing treatment were terminated prematurely owing to concern over possible hepatotoxic effects. ${ }^{17}$ Troglitazone was not approved as a study drug in some locations that had enrolled American Indian participants. The DPP preferentially enrolled individuals at high risk of developing T2DM: overall, $45 \%$ of participants were from high-risk ethnic groups, and $20 \%$ were aged $60-85$ years at baseline.

The DPP intensive lifestyle intervention was intended to achieve a 7\% loss in body weight over 24 weeks. Participants were instructed to perform 150 min moderate-intensity physical activity (such as brisk walking) per week and to eat a low-fat, reduced-calorie diet. They also received one-to-one counseling to aid these changes in behavior that was continued (generally monthly) after the initial 24-week period to help maintain weight loss and activity levels. The lifestyle-intervention group achieved a mean weight loss of $7 \%$ (an average of $7.0 \mathrm{~kg}$ ) within the first year, and had an overall mean weight loss of $5.6 \%$ (an average of $5.6 \mathrm{~kg}$ ) during followup (mean duration 2.8 years); the physical activity goals were met by $74 \%$ of participants in the first 24 weeks of the study.

The DPP lifestyle intervention was associated with a $58 \%$ reduction in the incidence of diabetes, compared with placebo plus standard lifestyle recommendations. ${ }^{19}$ Weight loss was the predominant predictor of reduced diabetes incidence, with a $16 \%$ reduction in risk per kilogram of weight lost. ${ }^{20}$ However, participants who achieved their exercise goals but not their weight loss goals also experienced some reduction in diabetes risk (44\%, compared with placebo-treated individuals). The effectiveness of the DPP lifestyle intervention was similar in all ethnic groups and both sexes, and was greatest in older participants (age 60-85 years). 21 Changes in physical activity and diet (primarily, a reduced calorie intake from fat) predicted weight loss, and weight loss, in turn, was associated with a reduced risk of developing T2DM. Interventions to reduce diabetes risk in overweight or obese individuals should, therefore, primarily aim to induce weight loss. 
Studies of diabetes prevention in Japan ${ }^{22}$ and India ${ }^{23}$ also confirmed the benefit of lifestylemodification programs. Even with quite modest weight losses $(0-2 \mathrm{~kg})$, risk reductions of $67 \%$ and $28 \%$ were achieved in the Japanese and Indian studies, respectively. The differences in study design and participant characteristics make direct comparisons difficult, but these findings do seem to support the effectiveness of lifestyle modification across a broad range of ethnic groups and cultures.

\section{Bariatric surgery}

Bariatric surgery can effectively reverse or prevent T2DM. Pories et al. ${ }^{24-26}$ noted the marked effect of weight-reduction surgery on diabetes. Although their data were retrospectively reviewed, they reported an annual diabetes incidence of $4.5 \%$ in the control group, compared with only $1.0 \%$ in the surgically treated group. In a prospective study of individuals with impaired glucose tolerance and severe obesity, diabetes incidence rates were 4.7 cases per 100 person-years of follow-up in the untreated (control) group, compared with 0.15 cases per 100 person-years in patients who underwent bariatric surgery. 27

The Swedish Obese Subjects (SOS) Study was a prospective trial of more than 2,000 individuals who underwent a variety of surgical procedures (most commonly, vertical banded gastroplasty) and matched controls who received standard care. ${ }^{28}$ The odds ratio for diabetes in the surgically treated group was 0.14 (95\% CI $0.08-0.24)$ at 2 years and 0.25 (95\% CI $0.17-$ 0.38 ) at 10 years of follow-up. As expected, the incidence of diabetes was related to the amount of weight lost. Of participants who lost more than $12 \%$ of their initial body weight none developed diabetes, in contrast to a 2-year diabetes incidence of $7 \%$ in those with a $2 \%$ weight loss, and $9 \%$ in those who gained weight. ${ }^{29}$ Other studies showed that laparoscopic adjustable gastric banding in patients with established diabetes resulted in remission of diabetes in $64 \%$ and major improvements in glycemic control in another $26 \%,{ }^{30}$ which suggests that this surgery could also successfully prevent diabetes.

In a randomized, controlled trial of gastric banding, diabetes remission was reported in $73 \%$ of the surgically treated patients, compared with $13 \%$ of recipients of conventional therapy. 31 Biliopancreatic diversion, the bariatric procedure that results in the most dramatic and sustained weight loss, has also been reported to normalize glucose tolerance in obese patients with either impaired glucose tolerance or established diabetes. ${ }^{32}$ Glucose tolerance has been reported to improve rapidly after bariatric surgery, even before much weight has been lost; neurohormonal mechanisms are reported to be involved. 33

\section{STUDIES OF PHARMACOLOGIC INTERVENTIONS}

Various pharmacologic agents have been studied (Table 2), including drugs commonly used to treat established diabetes (metformin, acarbose, thiazolidinediones) and a weight-loss drug (orlistat). ${ }^{34}$

\section{Metformin}

The biguanide, metformin, is the most thoroughly studied drug used for diabetes prevention. By far the largest group of metformin-treated patients studied was included in the DPP, in which high-risk individuals with impaired glucose tolerance were randomly allocated $850 \mathrm{mg}$ metformin twice daily $(n=1,073)$ or placebo $(n=1,082)$. Mean follow-up was 2.8 years. Adherence to metformin was excellent ${ }^{35}$ and, despite the anticipated gastrointestinal side effects and twice-daily dosing regimen, metformin reduced the risk of developing diabetes by $31 \%$ compared with placebo. Metformin was most effective in individuals whose baseline BMI was higher than $35 \mathrm{~kg} / \mathrm{m}^{2}$, in whom it reduced the incidence of diabetes by approximately $50 \%$. Metformin had little beneficial effect in older participants (those aged 60-85 years). An average 
$1.7 \mathrm{~kg}$ weight loss was reported in the metformin group, compared with a $0.3 \mathrm{~kg}$ weight gain in the placebo group; weight loss explained $64 \%$ of the beneficial effect of metformin on diabetes risk. Favorable changes in insulin sensitivity and in secretion of pro insulin also contributed to the decreased diabetes risk seen in metformin-treated patients. ${ }^{36}$

Two smaller studies in India ${ }^{23}$ and in $\mathrm{China}^{37}$ also reported similar reductions in diabetes risk with $250 \mathrm{mg}$ of metformin administered two or three times daily. The excellent safety record, long experience with this drug in the treatment of diabetes and low cost of metformin make it an attractive option for diabetes prevention.

\section{Thiazolidinediones}

The insulin-sensitizing properties of the thia-zolidinediones have generated enthusiasm for their role in diabetes prevention. Troglitazone reduced the development of diabetes by around $50 \%$ over 2.5 years in a group of women with prior gestational diabetes ( $60 \%$ also had impaired glucose tolerance at baseline). ${ }^{38}$ Troglitazone administration in the DPP was discontinued prematurely owing to its hepatotoxic effects, but during the average 0.9 years of exposure, diabetes incidence was reduced by $75 \% .39$ Administration of rosiglitazone over 3 years has since been studied in 5,269 patients with impaired fasting glucose, impaired glucose tolerance, or both. ${ }^{40}$ The incidence of diabetes was reduced by $62 \%$, and $50 \%$ of rosiglitazonetreated patients reverted to normoglycemia (compared with $30 \%$ of placebo-treated patients).

Like metformin, rosiglitazone seems to be most effective in individuals with a high BMI (rosiglitazone confers risk reductions of $40 \%$ for BMI $<28 \mathrm{~kg} / \mathrm{m}^{2}$ and $68 \%$ for BMI $>33 \mathrm{~kg}$ / $\mathrm{m}^{2}$ ). Notable side effects, including weight gain (rosiglitazone-treated patients gained $2.2 \mathrm{~kg}$ more than placebo-treated patients) and edema were observed, as they are when rosiglitazone is used to treat established diabetes. The frequency of congestive heart failure was also increased in the rosiglitazone group (hazard ratio 7.03, 95\% CI 1.60-30.9), but there were few cases in this generally healthy population. Questions have been raised about potential cardiovascular toxic effects of rosiglitazone and this issue remains to be resolved. ${ }^{41}$ Additional concerns about rosiglitazone include one report of an increased rate of osteoporotic fracture among diabetic women treated with this drug. ${ }^{42}$ These concerns might indicate an unacceptable risk-to-benefit ratio for thiazolidinediones, particularly rosiglitazone, in diabetes prevention. ${ }^{43,44}$

\section{Acarbose}

The $\alpha$-glucosidase inhibitor, acarbose, has been studied in a number of clinical trials. 37,45 , 46 Most notably, the STOP-NIDDM (Study to Prevent Non-Insulin-Dependent Diabetes Mellitus) trial ${ }^{47}$ reported that acarbose treatment was associated with a $25 \%$ reduction in the incidence of diabetes in a large $(n=1,429)$, cohort of high-risk individuals with impaired glucose tolerance. Approximately one-quarter of the cohort (including $31 \%$ of the acarbose group) did not complete the study, however, with the consequence that this estimate of the magnitude of acarbose's effect on diabetes incidence might be unreliable. The particularly high drop-out rate in acarbose-treated patients was attributed to its well-known gastrointestinal side effects. Acarbose might, therefore, have limited value for diabetes prevention in general practice.

\section{Weight-loss agents}

Weight-loss agents have been studied in small numbers of individuals with impaired fasting glucose, impaired glucose tolerance, or both. Orlistat, an intestinal lipase inhibitor, reduced the development of diabetes by $40-52 \%$ in obese people with impaired glucose tolerance. ${ }^{48,}$

49 The effect of this drug on diabetes might be attributable to weight loss, which was greater than that achieved with lifestyle change alone. The drop-out rate approached 50\%, which 
suggests that this therapy has limited acceptability, and potentially makes the estimates of the size of treatment effects unreliable.

\section{Other drugs}

Following post hoc analyses that suggested angiotensin-converting-enzyme inhibitors might reduce diabetes risk, ${ }^{50}$ ramipril was studied for diabetes prevention in the DREAM (Diabetes Reduction Assessment with Ramipril and Rosiglitazone Medication) trial. ${ }^{51}$ The hazard ratio for developing diabetes was 0.91 (95\% CI 0.80-1.03). There was no additive effect of therapy in participants who were randomly allocated both ramipril and rosiglitazone.

A post hoc analysis of data from a study of coronary artery disease prevention suggested that statins might have diabetes-prevention benefits, 52 but this finding has not been replicated ${ }^{53-}$ 55 and the hypothesis has not been tested in a randomized clinical trial.

Preliminary investigation of incretin-based therapies (e.g. exenatide and dipeptidyl peptidase IV inhibitors) suggests these agents not only enhance insulin secretion, but also promote $\beta$-cell proliferation. ${ }^{56}$ These agents might, therefore, be suitable for diabetes prevention, but clinical trials will be needed to address this hypothesis.

\section{MECHANISMS OF DIABETES PREVENTION}

In the DPP, both the intensive lifestyle intervention and metformin increased insulin sensitivity. 57 Intensive lifestyle intervention was more effective than metformin in slowing progression to diabetes, partly because lifestyle modification gave greater improvements in insulin sensitivity and $\beta$-cell function. ${ }^{57}$ Similarly, in the DPP ${ }^{39}$ and TRIPOD (Troglitazone in Prevention of Diabetes) ${ }^{38}$ studies, improvements in both insulin sensitivity and $\beta$-cell function explained the observed reduction in progression to diabetes seen with troglitazone. Data on the mechanistic effects of rosiglitazone in the DREAM study are not yet available. ${ }^{40}$

\section{Prevention versus masking of incident diabetes}

Whether the antidiabetic medications used for prevention truly prevent disease development or merely mask its presence is unclear. This issue has been addressed most directly in the DPP, in which a formal 1-2-week washout period was employed following completion of the initial randomized, double-blind trial. ${ }^{58}$ When an oral glucose-tolerance test (OGTT) was performed after washout, diabetes was more frequently diagnosed in metformin recipients than in placebo recipients (hazard ratio 1.49, 95\% CI 0.93-2.38, $P=0.098$ ). Even when these new cases of diabetes were included, however, the overall incidence of diabetes was still reduced by $25 \%$ in metformin-treated patients, which suggests that the benefit of metformin was not limited to its acute pharmacologic effects. Similar results were noted with acarbose in the STOP-NIDDM trial. $^{47}$

In the TRIPOD study of women with a history of gestational diabetes, 84 women were followed up for less than 1 year after drug discontinuation, during which time seven new cases of diabetes developed. Six of these cases occurred in the former placebo group and one in the former troglitazone group. These findings were interpreted as evidence for a persistent drug effect. 38 By contrast, in the DPP, the diabetes incidence in the former troglitazone group was almost identical to that in the former placebo group after drug withdrawal, ${ }^{39}$ and remained so during 3 years of follow-up. Results of long-term follow-up of individuals treated with rosiglitazone in the DREAM trial have not yet been published. 


\section{Vascular outcomes}

Despite substantial evidence for the effectiveness of both lifestyle change and medications in the prevention of diabetes, few data are available on the delay or prevention of vascular outcomes of diabetes. Acarbose treatment in the STOP-NIDDM trial was associated with a $49 \%$ reduction in patients' relative risk of cardiovascular events (hazard ratio $0.51,95 \% \mathrm{CI}$ $0.28-0.95, P=0.03$ ), although the number of events was small. ${ }^{59}$ A reduction was also seen in the risk of hypertension (hazard ratio $0.66,95 \% \mathrm{CI} 0.49-0.89, P=0.006$ ). The other diabetesprevention trials that are large enough to detect differences in low-frequency events have not yet reported vascular outcomes. However, in the DPP, improvements in various risk factors for cardiovascular disease, including serum lipids, C-reactive protein and fibrinogen, were seen with intensive lifestyle intervention and, to a lesser extent, with metformin. ${ }^{60,61}$ The incidence of metabolic syndrome was reduced by $41 \%(P<0.001)$ in the lifestyle-modification group and by $17 \%(P=0.03)$ in the metformin group, compared with its incidence in the placebo group. ${ }^{62}$ Likewise, indices that reflect cardiac autonomic function (heart rate variability) and physical fitness (resting heart rate) were also improved in the intensive lifestyle intervention group. ${ }^{63}$ Extended follow-up of this cohort is underway, which will include assessments of measures of subclinical atherosclerosis and cardiovascular disease events. Fibrinolysis (i.e. levels of plasminogen activator inhibitor 1) was improved by lifestyle intervention in the Finnish DPS. ${ }^{64}$

\section{Insights from genetic studies}

Genetic analyses have provided insights into the determinants of diabetes risk and the interaction of genes with responses to specific preventive interventions. The role of TCF7L2 polymorphisms in the development of diabetes has been confirmed. Furthermore, the association between the TT allele of the single-nucleotide polymorphism rs7903146 and decreased $\beta$-cell function was established in the DPP cohort. ${ }^{65}$ Metformin and the DPP intensive lifestyle intervention both had protective effects against the development of diabetes in those with high-risk alleles (American Indian participants were not well represented, since some centers did not approve the genetics studies). Similar results with respect to diabetes risk, insulin secretion and the effects of lifestyle modification were also obtained in the Finnish DPS. 66

Several genes are associated with the risk of developing T2DM, including KCNJ11 (which encodes islet ATP-sensitive inward rectifier potassium channel 11, also known as Kir6.2) and the adjacent gene $A B C C 8$ (which encodes sulfonylurea receptor 1). Polymorphisms in $K C N J 11$ and $A B C C 8$, as well as the common Pro12Ala polymorphism of PPARG were shown to influence the risk of developing diabetes in the prospective Botnia study, 67 the Finnish DPS, 68 and the DPP 69,70 populations. The roles of these genetic polymorphisms need to be confirmed in prospective studies. In the DPP, PPARG polymorphisms seemed not to influence the effectiveness of troglitazone in improving insulin sensitivity. ${ }^{69}$

\section{Translational studies}

Clinical trials that proved the effectiveness of lifestyle interventions in reducing diabetes risk were designed from the outset as efficacy trials. As such, they were conducted in resourceintensive settings with limited consideration as to how the intervention strategies might be adapted for population-wide use. Studies to investigate strategies for translating the findings of these efficacy trials into the public-health arena are in their infancy. Published studies of diabetes-prevention strategies have targeted populations known to be at high risk of developing $\mathrm{T} 2 \mathrm{DM},{ }^{71}$ including selected ethnic groups ${ }^{\dagger 2-79}$ and individuals with prediabetes, ${ }^{80-83}$ although many of these studies preceded publication of the DPP and, therefore, were not based on DPP evidence. Most of the studies combine dietary and exercise strategies as the basis of their interventions and use quasiexperimental designs for evaluation. ${ }^{71}$ 
Some community-based studies that preceded randomized clinical trials such as the DPP and DPS focused on implementation of school-based programs designed to increase physical activity and improve health awareness in young people. ${ }^{74,75,78,79}$ Results of these studies were varied, but suggested that these interventions improved participants' knowledge about healthy lifestyle choices and increased their adoption of risk-reducing behaviors, although effects on diabetes development have not been reported.

Studies in adults have focused primarily on interventions that increase levels of physical activity and promote consumption of a healthy or a weight-loss diet. ${ }^{76,77,84-88}$ In general, improvements were seen in rates of physical activity and some groups experienced weight loss, but these changes generally did not reach significance because of the study designs used, including the lack of control groups.

The most dramatic evidence that the DPP lifestyle intervention can be translated into community settings is offered by the work of Ackermann et al. ${ }^{80}$ and Ackermann and Marrero, 81 who used the Young Men's Christian Association as a delivery site for strategies that implemented the DPP intensive lifestyle intervention. These same researchers studied a groupbased version of the DPP curriculum in a randomized clinical trial; they reported significant weight loss in the intervention group that was sustained at 12 months after the curriculum ended. In addition, Finland has implemented a national program to prevent T2DM. ${ }^{89}$ The FIND2D (Finnish Type 2 Diabetes Prevention Plan 2003-2007) is being conducted in five hospital districts and covers a population of 1.5 million people. Primary and occupational health-care providers are the agents of intervention. The effectiveness, feasibility, and costs of the program are being evaluated.

\section{COST-EFFECTIVENESS OF DIABETES PREVENTION}

Although intervention can clearly reduce the incidence of T2DM in well-funded research settings, can those who fund health-care services afford such programs in practical settings? The economic evaluation performed by the DPP Research Group concluded that the DPP interventions would be cost-effective from societal and health-system perspectives, with costs per case of T2DM prevented or per quality-adjusted life-year gained similar to or lower than those of many other well-accepted interventions. ${ }^{90}$ Another analysis, however, suggested that such programs are too expensive for widespread implementation. ${ }^{91}$ Much of the uncertainty and disagreement between these analyses derive from their different assumptions about rates of progression to diabetes and its complications, as well as their different analytic approaches. 92 This question is, therefore, difficult to resolve with currently available data.

In India, preventive lifestyle or metformin interventions were deemed cost-effective, ${ }^{93}$ and might similarly be cost-effective in other developing countries. A systematic review of T2DM prevention strategies published in 2006 concluded that the interventions were highly costeffective, but noted that this interpretation was based on very few studies. ${ }^{94}$ The cost-effective ness of genotyping for gene variants with emerging evidence of an increased risk of T2DM remains to be assessed.

\section{PREVENTION VERSUS EARLY DETECTION AND TREATMENT}

Debate prevails about whether resources (human and financial) would be better spent on T2DM prevention or on its early detection and treatment. Early detection is feasible through use of the same simple tests used in prevention programs, and could be done much more economically than attempting to prevent diabetes at the population level. Allocation of resources to intensive management of patients with newly diagnosed diabetes could be preferable to prevention. A major drawback of this approach, however, is that many people will have already developed macrovascular disease (and, rarely, microvascular disease) before diagnosis. Nonetheless, no 
data from clinical trials that have specifically compared prevention with early detection and intensive treatment have yet been reported.

\section{RECOMMENDATIONS FOR CLINICAL PRACTICE}

Application of the research findings requires identification of individuals who are at risk of developing diabetes. Screening for prediabetes should be considered in people aged 45 years and older, and for adults younger than 45 years who are overweight or obese and have additional risk factors, such as a family history of diabetes, prior gestational diabetes or belonging to a high-risk ethnic group. ${ }^{95}$ Measurement of fasting plasma glucose levels will identify individuals with impaired fasting glucose, but an OGTT is necessary to identify impaired glucose tolerance. Impaired fasting glucose and impaired glucose tolerance are considered to confer equivalent increases in risk for the development of diabetes; the combination of impaired fasting glucose and impaired glucose tolerance confers a greater increase in risk than either variable alone. ${ }^{96,97}$ Strategies that employ measurements of fasting glucose and routine clinical variables to identify high-risk individuals have been proposed and have the advantage of not requiring administration of an OGTT. ${ }^{98,99}$ Such management algorithms seem to be capable of discriminating between individuals with varying levels of diabetes risk in clinical practice.

Lifestyle modification, including a weight-loss goal of 5-10\% of initial weight and moderateintensity physical activity for $30 \mathrm{~min}$ per day, can provide the greatest reduction in diabetes risk and should be implemented for all individuals at risk for diabetes. This approach has an excellent safety profile and might also be the most cost-effective intervention. The role of pharmacologic agents is somewhat less clear. However, substantial evidence supports the use of metformin in patients who either fail to achieve these lifestyle goals or who have additional risk factors at baseline (such as both impaired fasting glucose and impaired glucose tolerance, a strong family history of diabetes, dyslipidemia, or hypertension), especially if they are younger than 60 years and have a BMI of $35 \mathrm{~kg} / \mathrm{m}$ or greater. 2,96 Acarbose might be considered for these patients, but it is less well tolerated and more expensive than metformin, and requires three doses daily. Although effective, routine use of thiazolidinediones cannot currently be recommended because of their high cost and emerging evidence of adverse effects.

\section{CONCLUSIONS}

Tremendous progress has been made over the past decade in the identification of effective strategies to prevent or delay onset of T2DM. Important limitations in our current knowledge, however, include the lack of adequate data to determine whether these preventive approaches can slow the development of serious vascular complications of diabetes and ultimately reduce diabetes-related mortality. In addition, although important efforts in this regard are underway, the public-health messages implicit in diabetes risk and diabetes prevention remain underappreciated. Probably the greatest challenge continues to be in translating scientific discoveries into programs that are adequately funded and broadly available to individuals at high risk of developing diabetes.

\section{KEY POINTS}

- Type 2 diabetes mellitus is a common chronic disease that is responsible for enormous human and financial costs

- Lifestyle modification (i.e. modest weight loss and increased physical activity) is the most consistently effective approach to diabetes prevention

- Medications, including metformin, acarbose and thiazolidinediones, are effective therapies for preventing or delaying diabetes 
Interventions are effective across different ages and ethnic groups

- Application of these findings to public-health initiatives has been slow, at least partly because of their financial implications

\section{Supplementary Material}

Refer to Web version on PubMed Central for supplementary material.

\section{References}

1. Centers for Disease Control and Prevention. National diabetes fact sheet: general information and national estimates on diabetes in the United States, 2005. Atlanta, GA: US Department of Health and Human Services, Centers for Disease Control and Prevention; 2005.

2. Jensen CC, et al. Beta-cell function is a major contributor to oral glucose tolerance in high-risk relatives of four ethnic groups in the US. Diabetes 2002;51:2170-2178. [PubMed: 12086947]

3. Weyer $\mathrm{C}$, et al. The natural history of insulin secretory dysfunction and insulin resistance in the pathogenesis of type 2 diabetes mellitus. J Clin Invest 1999;104:787-794. [PubMed: 10491414]

4. Kahn SE. The relative contributions of insulin resistance and beta-cell dysfunction to the pathophysiology of Type 2 diabetes. Diabetologia 2003;46:3-19. [PubMed: 12637977]

5. Jarrett RJ, et al. Worsening to diabetes in men with impaired glucose tolerance ("borderline diabetes"). Diabetologia 1979;16:25-30. [PubMed: 761734]

6. Knowler WC, et al. Preventing non-insulin-dependent diabetes. Diabetes 1995;44:483-488. [PubMed: 7729603]

7. Keen H, et al. The ten-year follow-up of the Bedford survey (1962-1972): glucose tolerance and diabetes. Diabetologia 1982;22:73-78. [PubMed: 7060852]

8. Sartor G, et al. Ten-year follow-up of subjects with impaired glucose tolerance: prevention of diabetes by tolbutamide and diet regulation. Diabetes 1980;29:41-49. [PubMed: 7380107]

9. Knowler WC, et al. Glucose tolerance and mortality, including a substudy of tolbutamide treatment. Diabetologia 1997;40:680-686. [PubMed: 9222648]

10. Goede P, et al. Effect of a multifactorial intervention on mortality in type 2 diabetes. N Engl J Med 2008;358:580-591. [PubMed: 18256393]

11. Nathan DM, et al. Intensive diabetes treatment and cardiovascular disease in patients with type 1 diabetes. N Engl J Med 2005;353:2643-2653. [PubMed: 16371630]

12. Norris SL, et al. Long-term non-pharmacological weight loss interventions for adults with prediabetes. Cochrane Database of Systematic Reviews 2005. 2005;(Issue 1)Art. No.: CD005270

13. Pan XR, et al. Effects of diet and exercise in preventing NIDDM in people with impaired glucose tolerance: the Da Qing IGT and Diabetes Study. Diabetes Care 1997;20:537-544. [PubMed: 9096977]

14. Tuomilehto J, et al. Prevention of type 2 diabetes mellitus by changes in lifestyle among subjects with impaired glucose tolerance. N Engl J Med 2001;344:1343-1350. [PubMed: 11333990]

15. Laaksonen DE, et al. Physical activity in the prevention of type 2 diabetes: the Finnish diabetes prevention study. Diabetes 2005;54:158-165. [PubMed: 15616024]

16. Lindström J, et al. Sustained reduction in the incidence of type 2 diabetes by lifestyle intervention: follow-up of the Finnish Diabetes Prevention Study. Lancet 2006;368:1673-1679. [PubMed: 17098085]

17. The Diabetes Prevention Program Research Group. Design and methods for a clinical trial in the prevention of type 2 diabetes. Diabetes Care 1999;22:623-634. [PubMed: 10189543]

18. The Diabetes Prevention Program Research Group. Baseline characteristics of the randomized cohort. Diabetes Care 2000;23:1619-1629. [PubMed: 11092283]

19. Knowler WC, et al. the Diabetes Prevention Program Research Group. Reduction in the incidence of type 2 diabetes with lifestyle intervention or metformin. N Engl J Med 2002;346:393-403. [PubMed: 11832527] 
20. Hamman RF, et al. Effect of weight loss with lifestyle intervention on risk of diabetes. Diabetes Care 2006;29:2102-2107. [PubMed: 16936160]

21. The Diabetes Prevention Program Research Group. The influence of age on the effects of lifestyle modification and metformin in prevention of diabetes. J Gerontol A Biol Sci Med Sci 2006;61:10751081. [PubMed: 17077202]

22. Kosaka K, et al. Prevention of type 2 diabetes by lifestyle intervention: a Japanese trial in IGT males. Diabetes Res Clin Pract 2005;67:152-162. [PubMed: 15649575]

23. Ramachandran A, et al. The Indian Diabetes Prevention Programme shows that lifestyle modification and metformin prevent type 2 diabetes in Asian Indian subjects with impaired glucose tolerance (IDPP-1). Diabetologia 2006;49:289-297. [PubMed: 16391903]

24. Pories WJ, et al. Is type II diabetes mellitus (NIDDM) a surgical disease? Ann Surg 1992;215:633642. [PubMed: 1632685]

25. Pories WJ, et al. Surgical treatment of obesity and its effect on diabetes: 10-y follow-up. Am J Clin Nutr 1992;55(Suppl):S582-S585.

26. Pories WJ, et al. Who would have thought it? An operation proves to be the most effective therapy for adult-onset diabetes mellitus. Ann Surg 1995;222:339-350. [PubMed: 7677463]

27. Long SD, et al. Weight loss in severely obese subjects prevents the progression of impaired glucose tolerance to type II diabetes: a longitudinal interventional study. Diabetes Care 1994;17:372-375. [PubMed: 8062602]

28. Sjöström L, et al. Lifestyle, diabetes, and cardiovascular risk factors 10 years after bariatric surgery. N Engl J Med 2004;351:2683-2693. [PubMed: 15616203]

29. Sjöström, L. Surgical treatment of obesity: an overview of the results from the SOS study. In: Bray, GA.; Bouchard, C., editors. Handbook of Obesity: Clinical applications. New York: Marcel Dekker; 2004.

30. Dixon AF, et al. Laparoscopic adjustable gastric banding induces prolonged satiety: a randomized blind crossover study. J Clin Endocrinol Metab 2005;90:813-819. [PubMed: 15585553]

31. Dixon JB, et al. Adjustable gastric banding and conventional therapy for type 2 diabetes: a randomized controlled trial. JAMA 2008;299:316-323. [PubMed: 18212316]

32. Mari A, et al. Restoration of normal glucose tolerance in severely obese patients after bilio-pancreatic diversion: role of insulin sensitivity and beta cell function. Diabetologia 2006;49:2136-2143. [PubMed: 16819611]

33. Rubino F, et al. The early effect of the Roux-en-Y gastric bypass on hormones involved in body weight regulation and glucose metabolism. Ann Surg 2004;240:236-242. [PubMed: 15273546]

34. Gillies CL, et al. Pharmacological and lifestyle interventions to prevent or delay type 2 diabetes in people with impaired glucose tolerance: systematic review and meta-analysis. BMJ 2007;334:299. [PubMed: 17237299]

35. Walker EA, et al. Adherence to preventive medications: predictors and outcomes in the Diabetes Prevention Program. Diabetes Care 2006;29:1997-2002. [PubMed: 16936143]

36. The Diabetes Prevention Program Research Group. Factors associated with diabetes onset during metformin versus placebo therapy in the Diabetes Prevention Program. Diabetes 2007;56:11531159. [PubMed: 17395752]

37. Yang W, et al. The preventive effect of acarbose and metformin on the progression to diabetes mellitus in the IGT population: a 3-year multicenter prospective study. Chin J Endocrinol Metab 2001;17:131136.

38. Buchanan TA, et al. Preservation of pancreatic beta-cell function and prevention of type 2 diabetes by pharmacological treatment of insulin resistance in high-risk Hispanic women. Diabetes 2002;51:2796-2803. [PubMed: 12196473]

39. The Diabetes Prevention Program Research Group. Prevention of type 2 diabetes with troglitazone in the Diabetes Prevention Program. Diabetes 2005;54:1150-1156. [PubMed: 15793255]

40. Gerstein HC, et al. DREAM (Diabetes Reduction Assessment with Ramipril and Rosiglitazone Medication) Trial Investigators. Effect of rosiglitazone on the frequency of diabetes in patients with impaired glucose tolerance or impaired fasting glucose: a randomised controlled trial. Lancet 2006;368:1096-1105. [PubMed: 16997664] 
41. Nissen SE, Wolski K. Effect of rosiglitazone on the risk of myocardial infarction and death from cardiovascular causes. N Engl J Med 2007;356:2457-2471. [PubMed: 17517853]

42. Kahn SE, et al. Glycemic durability of rosiglitazone, metformin, or glyburide monotherapy. N Engl J Med 2006;355:2427-2443. [PubMed: 17145742]

43. American Diabetes Association. Prevention or delay of type 2 diabetes. Diabetes Care 2004;27:S47S54. [PubMed: 14693925]

44. Nathan DM, Berkwits M. Trials that matter: rosiglitazone, ramipril, and the prevention of type 2 diabetes. Ann Intern Med 2007;146:461-463. [PubMed: 17371892]

45. Holman RR, et al. Results from the early diabetes intervention trials [abstract]. Diabetes 2007;52:A16.

46. Pan CY, et al. Efficacy of acarbose in Chinese subjects with impaired glucose tolerance. Diabetes Res Clin Pract 2003;61:183-190. [PubMed: 12965108]

47. Chiasson JL, et al. Acarbose for prevention of type 2 diabetes mellitus: the STOP-NIDDM randomised trial. Lancet 2002;359:2072-2077. [PubMed: 12086760]

48. Torgerson JS, et al. XENical in the prevention of diabetes in obese subjects (XENDOS) study: a randomized study of orlistat as an adjunct to lifestyle changes for the prevention of type 2 diabetes in obese patients. Diabetes Care 2004;27:155-161. [PubMed: 14693982]

49. Heymsfield SB, et al. Effects of weight loss with orlistat on glucose tolerance and progression to type 2 diabetes in obese adults. Arch Intern Med 2000;160:1321-1326. [PubMed: 10809036]

50. Yusuf S, et al. Ramipril and the development of diabetes. JAMA 2001;286:1882-1885. [PubMed: 11597291]

51. Bosch J, et al. DREAM trial investigators. Effect of ramipril on the incidence of diabetes. N Engl J Med 2006;355:1551-1562. [PubMed: 16980380]

52. Freeman DJ, et al. Pravastatin and the development of diabetes mellitus: evidence for a protective treatment effect in the West of Scotland Coronary Prevention Study. Circulation 2001;103:357-362. [PubMed: 11157685]

53. Collins R, et al. MRC/BHF Heart Protection Study of cholesterol-lowering with simvastatin in 5,963 people with diabetes: a randomised placebo-controlled trial. Lancet 2003;361:2005-2016. [PubMed: 12814710]

54. Keech A, et al. Secondary prevention of cardiovascular events with long-term pravastatin in patients with diabetes or impaired fasting glucose: results from the LIPID trial. Diabetes Care 2003;26:27132721. [PubMed: 14514569]

55. Sever PS, et al. Prevention of coronary and stroke events with atorvastatin in hypertensive patients who have average or lower-than-average cholesterol concentrations, in the Anglo-Scandinavian Cardiac Outcomes Trial-Lipid Lowering Arm (ASCOT-LLA): a multicentre randomised controlled trial. Drugs 2004;64:43-60. [PubMed: 15765890]

56. Meece J. Pancreatic islet dysfunction in type 2 diabetes: a rational target for incretin-based therapies. Curr Med Res Opin 2007;23:933-944. [PubMed: 17407650]

57. The Diabetes Prevention Program Research Group. Role of insulin secretion and sensitivity in the evolution of type 2 diabetes in the diabetes prevention program: effects of lifestyle intervention and metformin. Diabetes 2005;54:2404-2414. [PubMed: 16046308]

58. The Diabetes Prevention Program Research Group. Effects of withdrawal from metformin on the development of diabetes in the diabetes prevention program. Diabetes Care 2003;26:977-980. [PubMed: 12663559]

59. Chiasson JL, et al. Acarbose treatment and the risk of cardiovascular disease and hypertension in patients with impaired glucose tolerance: the STOP-NIDDM Trial. JAMA 2003;290:486-494. [PubMed: 12876091]

60. The Diabetes Prevention Program Research Group. Impact of intensive lifestyle and metformin therapy on cardiovascular disease risk factors in the diabetes prevention program. Diabetes Care 2005;28:888-894. [PubMed: 15793191]

61. The Diabetes Prevention Program Research Group. Intensive lifestyle intervention or metformin on inflammation and coagulation in participants with impaired glucose tolerance. Diabetes 2005;54:1566-1572. [PubMed: 15855347] 
62. The Diabetes Prevention Program Research Group. The effect of metformin and intensive lifestyle intervention on the metabolic syndrome: the Diabetes Prevention Program randomized trial. Ann Intern Med 2005;142:611-619. [PubMed: 15838067]

63. Carnethon MR, et al. for the Diabetes Prevention Program Research Group. The association among autonomic nervous system function, incident diabetes, and intervention arm in the Diabetes Prevention Program. Diabetes Care 2006;29:914-919. [PubMed: 16567837]

64. Hämäläinen H, et al. Improved fibrinolysis by an intensive lifestyle intervention in subjects with impaired glucose tolerance: the Finnish Diabetes Prevention Study. Diabetologia 2005;48:2248 2253. [PubMed: 16205886]

65. Florez JC, et al. TCF7L2 polymorphisms and progression to diabetes in the Diabetes Prevention Program. N Engl J Med 2006;355:241-250. [PubMed: 16855264]

66. Wang J, et al. Variants of transcription factor 7-like 2 (TCF7L2) gene predict conversion to type 2 diabetes in the Finnish Diabetes Prevention Study and are associated with impaired glucose regulation and impaired insulin secretion. Diabetologia 2007;50:1192-1200. [PubMed: 17437080]

67. Lyssenko V, et al. Genetic prediction of future type 2 diabetes. PLoS Med 2005;2:e345. [PubMed: 17570749]

68. Laukkanen O, et al. Polymorphisms of the SUR1 (ABCC8) and Kir6.2 (KCNJ11) genes predict the conversion from impaired glucose tolerance to type 2 diabetes: the Finnish Diabetes Prevention Study. J Clin Endocrinol Metab 2004;89:6286-6290. [PubMed: 15579791]

69. The Diabetes Prevention Program Research Group. Effects of the type 2 diabetes-associated PPARG P12A polymorphism on progression to diabetes and response to troglitazone. J Clin Endocrinol Metab 2007;92:1502-1509. [PubMed: 17213274]

70. Florez JC, et al. Type 2 diabetes-associated missense polymorphisms KCNJ11 E23K and ABCC8 A1369S influence progression to diabetes and response to interventions in the Diabetes Prevention Program. Diabetes 2007;56:531-536. [PubMed: 17259403]

71. Satterfield DW, et al. Community-based lifestyle interventions to prevent type 2 diabetes. Diabetes Care 2003;26:2643-2652. [PubMed: 12941733]

72. Cook VV, Hurley JS. Prevention of type 2 diabetes in childhood. Clin Pediatr (Phila) 1998;37:123129. [PubMed: 9492121]

73. Engelgau MM, et al. A project to reduce the burden of diabetes in the African-American community: project DIRECT. J Natl Med Assoc 1998;90:605-613. [PubMed: 9803725]

74. Holcomb JD, et al. Evaluation of Jump Into Action: a program to reduce the risk of non-insulin dependent diabetes mellitus in school children on the Texas-Mexico border. J Sch Health 1998;68:282-288. [PubMed: 9779403]

75. Marlow E, et al. STOP diabetes! An educational model for Native American adolescents in the prevention of diabetes. Diabetes Educ 1998;24:441-450. [PubMed: 9830948]

76. Mau MK, et al. Mediators of lifestyle behavior change in native Hawaiians: initial findings from the Native Hawaiian Diabetes Intervention Program. Diabetes Care 2001;24:1770-1775. [PubMed: 11574440]

77. Narayan KM, et al. Randomized clinical trial of lifestyle interventions in Pima Indians: a pilot study. Diabet Med 1998;15:66-72. [PubMed: 9472866]

78. Teufel NI, Ritenbaugh CK. Development of a primary prevention program: insight gained in the Zuni Diabetes Prevention Program. Clin Pediatr (Phila) 1998;37:131-141. [PubMed: 9492122]

79. Trevino RP, et al. Bienestar: a diabetes risk-factor prevention program. J Sch Health 1998;68:62-67. [PubMed: 9571575]

80. Ackermann RT, et al. Translating the Diabetes Prevention Program into the community: the YMCA model [abstract]. Diabetes 2007;67:A42.

81. Ackermann RT, Marrero DG. Adapting the Diabetes Prevention Program lifestyle intervention for delivery in the community: the YMCA model. Diabetes Educ 2007;33:69-78. [PubMed: 17272794]

82. Ratner R. Community-based identification of pre-diabetes: efficient screening techniques for implementation of diabetes prevention strategies [abstract]. Diabetes 2004;61:A290.

83. Seidel MC, et al. Translating the Diabetes Prevention Program (DPP) in an urban underserved community: long term sustainability of positive clinical outcomes [abstract]. Diabetes 2007;67:A42. 
84. Bjärås G, et al. Walking campaign: a model for developing participation in physical activity? Experiences from three campaign periods of the Stockholm Diabetes Prevention Program (SDPP). Patient Educ Couns 2001;42:9-14. [PubMed: 11080601]

85. Daniel M, et al. Effectiveness of community-directed diabetes prevention and control in a rural Aboriginal population in British Columbia, Canada. Soc Sci Med 1999;48:815-832. [PubMed: 10190643]

86. Rowley KG, et al. Reduced prevalence of impaired glucose tolerance and no change in prevalence of diabetes despite increasing BMI among Aboriginal people from a group of remote homeland communities. Diabetes Care 2000;23:898-904. [PubMed: 10895838]

87. Simmons D, et al. A pilot diabetes awareness and exercise programme in a multiethnic workforce. NZ Med J 1996;109:373-376.

88. Simmons D, et al. A pilot urban church-based programme to reduce risk factors for diabetes among Western Samoans in New Zealand. Diabet Med 1998;15:136-142. [PubMed: 9507914]

89. Saaristo T, et al. National type 2 diabetes prevention programme in Finland: FIN-D2D. Int J Circumpolar Health 2007;66:101-112. [PubMed: 17515250]

90. Herman WH, et al. for the Diabetes Prevention Program Research Group. The cost-effectiveness of lifestyle modification or metformin in preventing type 2 diabetes in adults with impaired glucose tolerance. Ann Intern Med 2005;142:323-332. [PubMed: 15738451]

91. Eddy DM, et al. Clinical outcomes and cost-effectiveness of strategies for managing people at high risk for diabetes. Ann Intern Med 2005;143:251-264. [PubMed: 16103469]

92. Engelgau MM. Trying to predict the future for people with diabetes: a tough but important task. Ann Intern Med 2005;143:301-302. [PubMed: 16103474]

93. Ramachandran A, et al. Cost-effectiveness of the interventions in the primary prevention of diabetes among Asian Indians: within-trial results of the Indian Diabetes Prevention Programme (IDPP). Diabetes Care 2007;30:2548-2552. [PubMed: 17670917]

94. Vijgen SM, et al. Cost effectiveness of preventive interventions in type 2 diabetes mellitus: a systematic literature review. Pharmacoeconomics 2006;24:425-441. [PubMed: 16706569]

95. American Diabetes Association. Standards of medical care in diabetes-2008. Diabetes Care 2008;31:S12-S54. [PubMed: 18165335]

96. Nathan DM, et al. Impaired fasting glucose and impaired glucose tolerance: implications for care. Diabetes Care 2007;30:753-759. [PubMed: 17327355]

97. Edelstein SL, et al. Predictors of progression from impaired glucose tolerance to NIDDM: an analysis of six prospective studies. Diabetes 1997;46:701-710. [PubMed: 9075814]

98. Stern MP, et al. Identification of persons at high risk for type 2 diabetes mellitus: do we need the oral glucose tolerance test? Ann Intern Med 2002;136:575-581. [PubMed: 11955025]

99. Wilson PW, et al. Prediction of incident diabetes mellitus in middle-aged adults: the Framingham Offspring Study. Arch Intern Med 2007;167:1068-1074. [PubMed: 17533210]

\section{Acknowledgments}

The authors thank G Trandafirescu and $\mathrm{H}$ Shamoon for their valuable assistance in the preparation of this manuscript. 


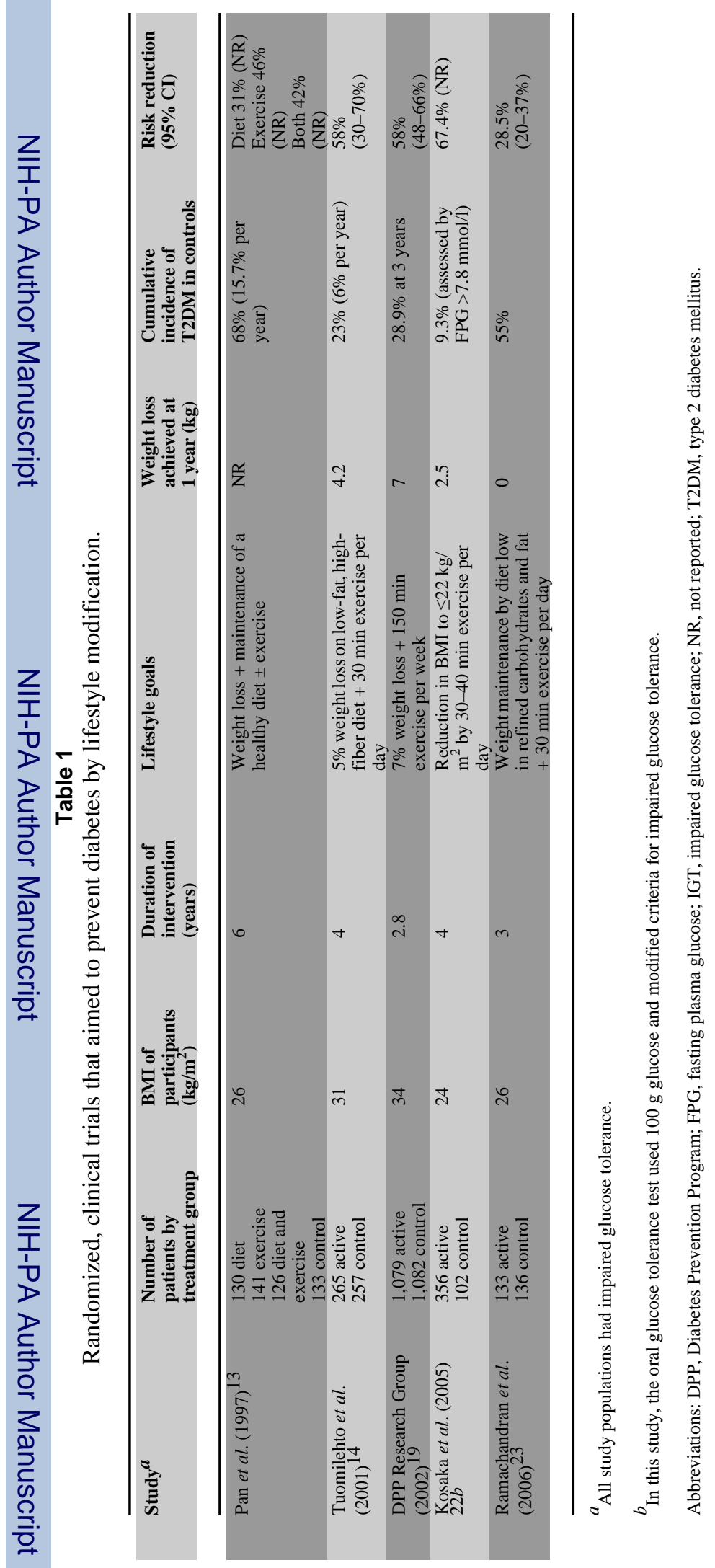

Nat Clin Pract Endocrinol Metab. Author manuscript; available in PMC 2008 October 27. 


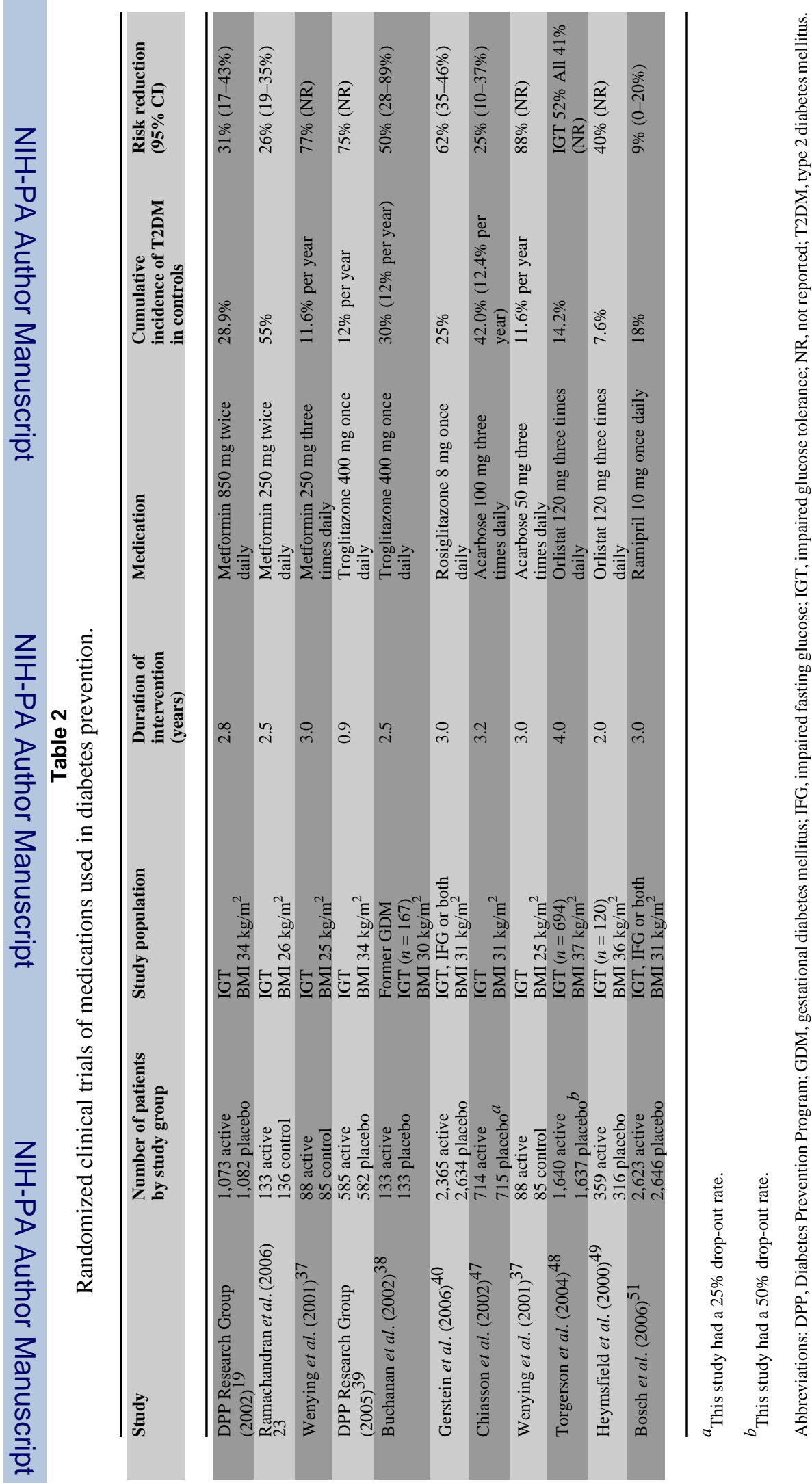

Nat Clin Pract Endocrinol Metab. Author manuscript; available in PMC 2008 October 27. 